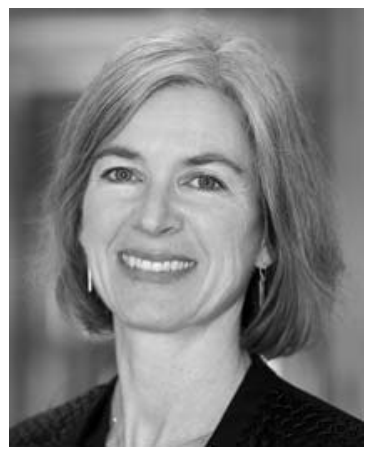

\title{
A Conversation with Jennifer Doudna
}

\author{
INTERVIEWER: JAN WITKOWSKI \\ Executive Director, Banbury Center at Cold Spring Harbor Laboratory
}

Jennifer Doudna is a Professor in the Department of Chemistry and the Department of Molecular and Cell Biology at the University of California-Berkeley.

Jan Witkowski: People know of you primarily through your work on the CRISPR-Cas9 system for genetic engineering. Can you go over the biology of the system and how you got involved in working on it?

Dr. Doudna: We started working on CRISPR (clustered regularly interspaced short palindromic repeats) biology about 10 years ago. A colleague of mine at Berkeley, Jillian Banfield, was doing research on bacterial communities and the viruses that infect them. She had noticed a lot of repetitive sequences in their genomic data and wondered if these were being used in the form of RNA molecules to protect the bacteria from viral infection. This led to our work with Emmanuelle Charpentier to understand the function of a particular protein called Cas9 (CRISPRassociated protein 9), which turns out to be an RNAguided DNA cutting enzyme. This is a great way for bacteria to fight viruses. It's an adaptive immune system. The bacteria acquire genetic material from viruses and insert them into these CRISPR sequences. They can transcribe the stored sequence into RNA, and then those RNA molecules can base-pair the matching viral DNAs sequences. They use RNA molecules to target the viral sequences and Cas9 cuts the viral DNA. About half of the sequenced bacterial genomes have one or more CRISPR loci in the genome.

Jan Witkowski: Why is it not more widespread?

Dr. Doudna: Bacteria have a lot of ways to avoid viruses. CRISPR systems operate in certain kinds of bacteria, perhaps in certain environments where they're particularly advantageous. Other bacteria simply might not need them because they have other ways of fighting the viruses they encounter.

Jan Witkowski: Bacteria have different enzymes depending on the type of CRISPR system, but Cas9 is the one that caught people's attention for genome engineering. Why is that particularly useful?

Dr. Doudna: It's programmable. It can be targeting using a short sequence of RNA that provides the base-pairing information to recognize DNA molecules with a matching or complementary sequence. Cas9 is also useful because the enzyme cuts both strands of double-stranded DNA.

Jan Witkowski: From an engineering point of view, how does it improve over other ways of making directed cuts in DNA systems, like TALENs (transcription activatorlike effector nucleases)?

Dr. Doudna: Introducing double-stranded breaks into a cell's genome can be a useful way to change the genetic sequence of those cells in a precise fashion, and over the years a number of technologies have been used. Back in the 1980s, Peter Dervan's lab, for example, came up with various chemical methods of cleverly targeting particular sequences. There also are various approaches where you can engineer proteins that have a particular DNA recognition property, enabling them to bind to a particular site in the genome and introduce a break.

The problem is that it's been really hard to make those other technologies widely applicable. In the case of proteins that recognize DNA, you have to engineer a new protein for each experiment. The nifty thing about Cas 9 is that the RNA does the programming, and it's fairly trivial to make a new strand of RNA that provides the targeting information. The protein stays the same in every experiment, and you change the specificity just by switching out the RNA.

Jan Witkowski: It reminds me of telomerase, in the way it prevents telomeres from being shortened with each division.

Dr. Doudna: There's an interesting parallel there. Telomerase also uses a guide RNA to template the extension of sequences at the end of the telomere, but this is not a polymerase. This is a protein that simply binds to DNA and then cuts it. Nonetheless, they both use RNA/DNA base-pairing to do that.

Jan Witkowski: It's fascinating what an important role RNA has played. When were the first small RNAs found? 
Dr. Doudna: In the 1990s. I remember Gary Ruvkun at Harvard, where I was a graduate student, telling me sometime in the early ' 90 s that they had found a gene called let-7 in the nematode Caenorhabditis elegans that encodes a small RNA. They had no idea what its function was, but they knew genetically that it was very important for the development of the worm. That was the first time I heard about a small RNA being functional.

Jan Witkowski: What are the steps in synthesizing the guide RNA? Does the enzyme just pick this up or do you have to wed the two?

Dr. Doudna: The beautiful thing about the system is that it has a natural ability for the protein and the RNA to come together. This is the work I did with Emmanuelle Charpentier's lab. In nature, Cas9 actually binds to two separate RNA molecules. One of them provides the DNAtargeting information, and the other provides the structural interaction with that targeting RNA to allow binding of the Cas9 protein. We figured out that we could actually re-engineer those two RNAs into a single guide RNA that provides both of those functions in the same molecule. It makes it a much simpler system for genome editing.

The way one applies this as a technology is to introduce the Cas 9 protein into cells either by introducing it with an encoded DNA molecule like a plasmid, or in the form of an RNA that encodes the protein, or even just as the protein itself. The guide RNA can be inserted using any of those same methods. The protein has a nuclear localization signal engineered into it, so it goes into the nucleus. Once the protein and the RNA are together in the cell, the affinity of binding between them is very specific and very high. Cas9 will actually find the guide RNA out of all the RNAs in the cell, bind to it, and use that for DNA recognition and cutting. In my lab, some of the early testing of this in mammalian cells was done by a firstyear graduate student. She's very good and very talented, but she was inexperienced. It showed us how straightforward and easy the technology is to use.

Jan Witkowski: It's relatively easy to do in cells and cultures. What are the complications when you try to do it in vivo?

Dr. Doudna: The major issue there is delivery. How do you get it into particular tissues? How do you get it into an organ as a therapeutic application? That's a challenge for any of these technologies. Hand-in-hand with that go the issues of efficacy and specificity. If we're using it as a human therapeutic, can we ensure that the editing that happens is accurate enough for that kind of application?

Jan Witkowski: What sort of applications has it been used for?
Dr. Doudna: There have been a number of reports using this in various animal models. Tyler Jacks' lab at MIT has done beautiful work where they target particular genes in mice and show that they can have a therapeutic benefit, or they can actually induce types of cancers that otherwise couldn't be studied very easily. You can use it either way in animal systems. Hans Clevers pioneered the use of organoids, where they can culture cells that become organ-like in a Petri dish. He's used the system to show that you can correct mutations that cause cystic fibrosis. People have also shown that you can take cells from patients that have sickle cell anemia or other genetic blood disorders and use the Cas 9 system to edit those cells and introduce corrective mutations. There's incredible potential for this. We just need to put the steps in place to figure out the delivery and safety issues.

With regard to using this technology in humans, the field is moving forward very quickly. It's likely we'll see clinical trials within the next three years in various human systems, probably in tissues that are amenable to introducing this kind of technology. I think hematopoietic cells, or the eye, or the liver, are the most likely initial targets. Beyond that, if clinical trials are initiated and if they go well, realistically it's still going to be at least 10 years before we could expect FDA approval. Still, when you realize that this technology is only a little over 2 years old, it's quite astounding to think that this is a real possibility.

Jan Witkowski: The enthusiasm about gene therapy in the 1980 s never really came to pass. To some people, just the words "gene therapy" raise Frankenstein-like images.

Dr. Doudna: The original gene therapy approaches were not using as precise a way to introduce information into genomes. They relied on viral integration where the virus decided where it was going, not the experimenter. That proved to be problematic. I think now we have a tool that really is highly specific. It's sort of a molecular scalpel that allows very precise cutting of the DNA. We do need to get a better handle on the repair process after the cut is made. Right now, for example, it's fairly easy to make knockouts of genes, to disrupt a sequence in a gene. It's harder to make a knockin of new genetic information. It can be done, but cells decide how to do it, not the experimenter. That's one of the areas of research that will be important for ensuring the safety of this technique. I think this is one reason there's interest in using hematopoietic stem cells. You can edit them, then verify and validate it in vitro before putting those cells back into a patient. I feel confident that the field will move in a direction where we have enough understanding of how it works and how to make sure it's accurate that it can be employed in other systems. 


\section{$\$_{\text {CSH }}^{\infty}$ Cold Spring Harbor Symposia SYMPOSIA On Quantitative Biology}

\section{A Conversation with Jennifer Doudna}

Cold Spring Harb Symp Quant Biol 2015 80: 314-315

Access the most recent version at doi:10.1101/sqb.2015.80.030072

\section{License}

Email Alerting Receive free email alerts when new articles cite this article - sign up in Service the box at the top right corner of the article or click here. 